\title{
Feasibility of Primary Prevention of Cardiovascular Diseases in Pakistan
}

\author{
Kalim uddin Aziz \\ Department of Paediatric Cardiology, National Institute of Cardiovascular Diseases, Karachi, Pakistan
}

Pakistan is a developing country and its health delivery system is not integrated, in fact it is upside down, i.e. emphasis is on tertiary care. District and tehsil hospitals and basic health units exist mostly on paper. Here, the data regarding escalating cardiovascular diseases (CVD) is compelling. ${ }^{1-2}$ Present situation has come about because of the inability to manage the epidemiological transformation of the population from rural to urban lifestyles. There is no debate about the imperative need of prevention. The question is: what type of preventive strategy is possible in Pakistan and to what effect? Poverty, lack of education, strong cultural biases, unplanned urbanisation and absence of health services in the community, school or workplace form a broad canvas of problems to overcome.

Primary prevention aims at reducing the risk factors by means of diet control, increasing physical activities, and prohibition of smoking and alcohol to a level required to reduce the prevalence of CVD. It can be implemented in a population or community or individual. This strategy requires sustained compliance for over a 10-year period to affect positive change. Risk factor modification requires changing the lifestyle of community or the entire population. Lifestyle modification involves change in one's diet from fatty saturated to unsaturated oils, vegetables, lentils, fish, chicken, and of salt to less than $5 \mathrm{~g} /$ day. It involves giving up of alcohol and smoking and sedentary habits, and adopting physically active habits, risk walk for $1 / 2$ hour three times per week. WHO and European guidelines provide the lower levels of risk factors for an individual or a country's risk of CVD., The means by which this can be achieved, is not a simple matter, rather it requires motivation to change. Motivation is generated by acquisition of knowledge regarding the risk factors associated with the causation from CVD; and the conviction that their modification will result in reduced risk of dying from heart attack and stroke. Motivation leads to generation of will to change.

Correspondence to: Prof. Kalim uddin Aziz, Department of Paediatric Cardiology, National Institute of Cardiovascular Diseases, Karachi

E-mail: azizkalim36@gmail.com

Received: February 27, 2020; Revised: July 07, 2020;

Accepted: July 13, 2020

DOI: https://doi.org/10.29271/jcpsp.2020.08.777
In this strategy, risk factors are identified and modified with medicines. This can be applied in an individual, population or a community. This approach requires funds to sustain it. A multinational global study has shown that this approach to be ineffective in low income countries and communities. ${ }^{5}$ This strategy can bea complement to primary prevention. Secondary prevention can also be applied in high risk individuals identified following cardiovascular event, a high-risk family or high-risk individuals discovered on community survey.

A meta-analysis of 36 studies conducted between 1972-2019 for primary prevention of CVD employed media, controlled screening before and after intervention, environmental changes and counselling. In 26 studies, the average net reduction expected / observed after 10 years of intervention was $0.65 \%$ risk reduction in CVD mortality. In other studies, there was a positive change in mortality, but it was not significant. ${ }^{6}$

The most successful community study, conducted in Karelia community in Finland, reported its results after 40 years of sustained community involvement, in which coronary artery prevalence was reduced by $84 \%$ as well as significant reduction of risk factors such as in men the smoking fell from 52.6 to $29.3 \%$, serum cholesterol from 6.77 to $5.44 \mathrm{~mm} / \mathrm{l}$, and systolic blood pressure from 147.1 to $133.5 \mathrm{mmHg}$. In women similarly smoking prevalence unchanged from 11.4 to $19.4 \%$, serum cholesterol from 6.69 to $5.3 \mathrm{~mm} / \mathrm{l}$, and systolic blood pressure from 149.2 to $129.1 \mathrm{mmHg}$. The reduction in risk factors and coronary artery disease occurred over successive years of sustained intervention.

Karelia study, conducted in a community of Karelia, identified three risk factors: smoking, high blood pressure and high cholesterol; and their reduction accounted for 69 percent of observed reduction in mortality due to coronary heart disease in men and $65 \%$ in women. The remaining risk factors accounted for rest of the reduced mortality. ${ }^{7-9}$

There are a few studies reported from developing countries as well. A well-structured study was performed on the population of three districts of Isfahan, one served as control and two in which primary prevention was implemented over a 7 -year period. The intervention involved introducing healthy diet, increased physical activity and tobacco control. Media school and community workplaces were targeted, meeting with the use of community leader lectures and healthy diet pamphlets. Significant reduction in serum cholesterol, blood pressure and BMI were reported nochange in diabetes. ${ }^{10}$ 
A population-based intervention was undertaken in women of Northern India, as five urban and five rural districts were selected. The purpose was to enhance knowledge and attitude regarding CVD and encourage vegetable diet and low fat. The mode of intervention was street plays / posters, and group discussions regarding knowledge about CVD. The purpose was to encourage use of low fat and high fiber diet. The interventions were delivered over six-month period. Knowledge regarding CVD was significantly increased, but no change in practice of using low fatand high fiber diet was noted. ${ }^{11}$

A cooperative study based in Ballabgarh district in Delhi, India and Depok districtin Indonesia has been reported. It was population-based study in which $25-35 \%$ population was accessed. Voluntary workers of the study group interacted with the existing health delivery infrastructure. There was a slight reduction in smoking and alcohol use; while, the use of vegetable and fruit also decreased. The health delivery infrastructure was found to be weak in Ballagarh; while in Depok, community health system was better, so that physical activities increased significantly as did obesity; while blood pressure and sugar levels decreased. ${ }^{12}$

A community-based, randomised controlled risk factor modification study, by Metroville Health Study (MHS), was undertaken between 1995-2000 in lower middle-class urban community (LMCUC) of Metroville, situated in the environs of Karachi, Pakistan. It targeted major risk factors; and the intervention was delivered in the household by trained social workers. Direct interaction was established by the study group and the community. At its core was the teaching hospital, National Institute of Cardiovascular Diseases (NICVD), where all the planning and implementation was undertaken in collaboration with the team from National Institute of Heart, Lung and Blood at Bethesda. The aim was to develop a model which could be implemented in all the LMCUC in the environs of all the teaching hospitals in Pakistan.

The MHS model was successfully implemented in the short term in LMCUC of the Metroville. The intervention was delivered in the households, diet was successfully modified, and awareness was increased remarkably in the short term. ${ }^{13}$ After five years of intervention, the results showed that awareness was enhanced, prevalence of smoking was reduced significantly, but no change was noted in blood pressure or BMI. The cholesterol level decreased in intervention group, but not significantly. Morbidity and mortality of CVD did not change. The prevalence in the community could not be ensured due to failure to establish a community centre empowered by the community. $^{14}$

Another intervention study was reported from Pakistan in 2007, conducted by international department of UK in collaboration with Heartfile - a website. The intervention was delivered in three districts of Lodhran; and a district in Rahim Yar Khan, which acted as control. The mode of intervention was by use of mass media, lectures, and pamphlets regarding the causes of CVD. The knowledge and attitudes, diet and physical activities were targeted. Lady health workers were trained and worked with basic health units in the districts. The study lasted for three years. Knowledge regarding CVD was increased and no change was noted in risk factors. ${ }^{15}$

The above experiences show that primary prevention reduces the prevalence of risk factors and mortality significantly; but the intervention has to be sustained at least for over a 10-year period. In developed countries, it has been implemented with success. When combined with secondary prevention with medicines, significant results in reducing mortality can be achieved in the short and long term. In the developed countries, the intervention team interacted with strong health delivery in the communities in schools and workplaces. The use of media, healthy smoke-free environment, and dietary controls showed success of intervention and long-term sustainability of intervention.

The studies reported from the developing countries show that risk factors were modified significantly when community health infrastructures were present and functioning. The studies reported from Pakistan, demonstrated that with the absence of functioning primary health entities, long term sustainability of intervention was not possible. The key to success is empowerment of the community and the perception that they own the intervention effort. The dietary and environmental controls, and access to the media are not consistently available. Mechanisms for community's empowerment need to be evolved, which are suitable to the cultural attitudes of the population. The lesson learnt is that without primary health delivery infrastructure, primary prevention is not feasible.

Rapid unplanned urbanisation has created a large high-risk population; and we, so far, have been unable or unwilling to prevent. Robust primary care infrastructure in village and urban communities, schools and workplace is crucial. Without clean water and efficient sanitation, primary prevention of non-communicable diseases shall remain problematic. Thus, the time to act is now!

\section{REFERENCES}

1. WHO impact of chronic disease in Pakistan. http//www. who.pnt/chp/cronic-disease-report/en/e, and low-CDC Global Health Pakistan. http//www.cdc.gov/globalhealth/ countries/Pakistan/default/htm

2. Piepoli MF, Hoes AW, Agewall S, Albus C, Boroton C, Catalan AE et al. European guide lines on cardiovascular diseases in clinical practice. European Heart J 2016; 37 (29):2315-81.

3. Prevention of cardiovascular disease guideline for assessment and management of cardiovascular risk. World health organization. 2007 ISBN 978924154717815.

4. Yusuf S, Islam Chow CK, Rangrajan S, Dagenais G, Dias R, Gupta $R$, et al. Use of secondary prevention drugs for cardio vascular disease in the community in high - income, middle - income countries (the pure study): A prospective epidemiologic survey. Lancet 2011; 378(9798):1231-40. Doi:10.1155/2011/161574.Epu2011aug26. 
5. Pennant M, Devenport C, Bayliss S Greenfield W, Marshal T, Hyde C. Community programs for the prevention of cardiovascular disease: A systematic review. Am J Epidemiol 2010; 172(5):501-516. doi: 10.1093/aje/kwq171.

6. Puska P, Salonen JT, Nissinen A, Tuomilehto J, Vartiainen E, Korhnonen $\mathrm{H}$, et al. Change in risk factors for coronary artery heart disease During 10 years of a community intervention Program. BMJ 1983; 287:1840-4. doi: http://doi.org/10.1136/bmj.287.6408.1840

7. Puska P. Success full prevention of non-communicable diseases: 25-year experience with North Karelia Project in Finland. Public Health Med 2002; 4(1):5-7.

8. Jousilahti P, Laatikainen T, Salomaa V, Vartiainen E, Puska P. 40-year CHD mortality trends and the role of risk factors, mortality decline. Global Heart 2016; 11(2):207-12. doi: 10.1016/j.gheart.2016.04.004.

9. Sarafzadegan N, Kelishade R, Ismaillzade A, Mohammadifard N, Rabiel K, Roohafza H, et al. Do life style interventions work in developing countries findings from Isfahan Heart program in Islamic republic of Iran. Bull World Health Organ 2009; 81(1):39-50. doi: 10.2471/blt.07. 049841
10. Panday RM, Agarwal A, Misra A, Vikram NK, Misra P, Day S, et al. Population based intervention for cardiovascular diseases related knowledge and behavior in Asian women. Indian Heart J 2013; 65(1):40-7. doi: 10.1016/j.ihj. 2012.12.019.

11. Krishnan A, Ekowati, Baridalyne N, Kurshmawardani N, Suhardi, Kapoor SK. Evaluation of community-based intervention of non-communicable diseases Indonesia. Health Promote Int I 2000; 26(3):276-89. doi: 10.1093/ heapro/daq067.

12. Aziz KH, Dennis B, Davis CE, Us K, Burke G, Manolio T, et al. Efficacy of CVD risk factor modification in a lower middleclass community in Pakistan. The Metroville Health study. Asia Pac J Public health 2000; 15(1):30-3. doi: 10.1177/ 101053950301500106.

13. Aziz K. A review of risk factors for primary prevention of cardiovascular disease in pakistan. Pak Armed Forces Med J 2017; 67 (suppl-2):S248-53.

14. Nishtar S, Badar A, Kamal M, Iqbal A, Bajwa A, Shah T, et al. The heart file; Lodhran CV prevention project. Promotion and Education 2007; 11(1):17-27. doi.org/10.1177/ 175797590701400103 